\title{
Response of alumina resistance to trace concentrations of acetone vapors at room temperature
}

\author{
Ján Ivančo*, Yuriy Halahovets*, Monika Benkovičová*, Matej Mičušík ${ }^{* *}$, \\ Jozef Kollár $^{* *}$, Dmytro Kostiuk* , Ana Hološ ${ }^{* *}$, Jaroslav Mosnáček ${ }^{* *}$
}

\begin{abstract}
The study demonstrates that resistivity of an alumina wafer is highly sensitive to trace concentrations of acetone vapors at room temperature. Though, a thermal pretreatment is necessary to precede the room-temperature sensing of acetone vapors, whilst the sensitiveness increases with the pretreatment temperature. This advocates the alumina being suitable for an adequate acetone sensor in the ppm range. A plausible mechanism of the response is discussed.
\end{abstract}

K e y w ords: gas sensor, chemiresistor, acetone, $\mathrm{Al}_{2} \mathrm{O}_{3}$, alumina

\section{Introduction}

The exhaled breath of a human contains a variety of volatile organic compounds (VOC); their presence and concentration could be suggestive of the health condition. For example, the background value of $0.76 \mathrm{ppmv}$ acetone vapors associated with a healthy person rises with a diabetes patient; the value of 1.7 [1] and even as high as 10 ppmv [2] were reported. A simple chemiresistor-inferred sensor capable to detect acetone vapors could facilitate the development of a personal instrument for daily monitoring of diabetes patients, thus providing an affordable non-invasive alternative to blood testing.

The fundamental structure of the chemiresistor consists of a gas-sensitive layer supported by an electrically insulating substrate, the former being the most frequently based on a semiconducting metal oxide [3-5]. The maximal sensing response occurs typically at elevated operating temperature up to several hundreds of degrees Celsius. The stoichiometry of compounds such as metal oxides is usually temperature-dependent and the stabilization of sensor transport properties at elevated operating temperature may request long start-up time of tenths of minutes. The lengthy onset can lead to a resistance drift with magnitude comparable to the gas-induced response. Therefore, the elevated operating temperature can be in general disadvantageous due to stability issues and high power consumption. A room-temperature operated sensor would apparently avoid drawbacks related to elevated operating temperature, yet such sensors have been merely intermittently reported (see eg [6-7] and remained a challenge.

Alumina has been commonly employed in the role of the supporting substrate for gas-sensitive films for its ex- cellent insulating properties and stability at high temperatures. In this study, we demonstrate that the supporting alumina itself can show a marked response to trace concentration of acetone vapors even at room temperature if subjected to a thermal pretreatment, and can be thereby classified as the virtually room-temperature sensor of acetone vapors.

\section{Experimental}

The alumina chips sized $2 \times 2 \mathrm{~mm}$ cut out from a 0.25 mm thick wafer (purity 99.0\%, Goodfellow Cambridge) were equipped with interdigital planar electrodes (distanced $50 \mu \mathrm{m}$ ) on the front side allowing the film resistivity characterization, and an integrated resistive heater incorporated on the back side. The electrodes (see the drawing sketched in Fig. 1) were made of $50 \mathrm{~nm} \mathrm{Ti} / 500$ $\mathrm{nm}$ Pt films. The chips were soldered into a commercial TO-4 socket.
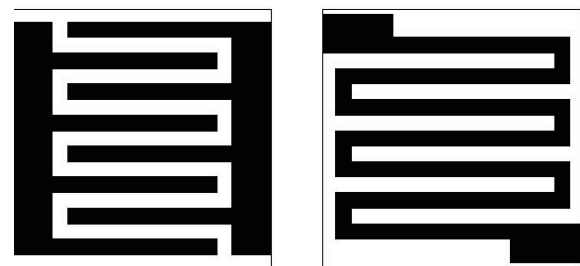

Fig. 1. Schematic drawing of interdigital electrodes and the resistive meander on the front and back sides (left and right images, respectively) of the $\mathrm{Al}_{2} \mathrm{O}_{3}$

The samples were annealed in the gas-sensing probing cell at temperatures up to $440^{\circ} \mathrm{C}$ in synthetic air, whilst

*Institute of Physics, Slovak Academy of Sciences, Dúbravská cesta 9, SK-845 11 Bratislava, Slovakia, jan.ivanco@savba.sk, **Institute of Polymers, Slovak Academy of Sciences, Dúbravská cesta 9, SK-845 41 Bratislava, Slovakia, 


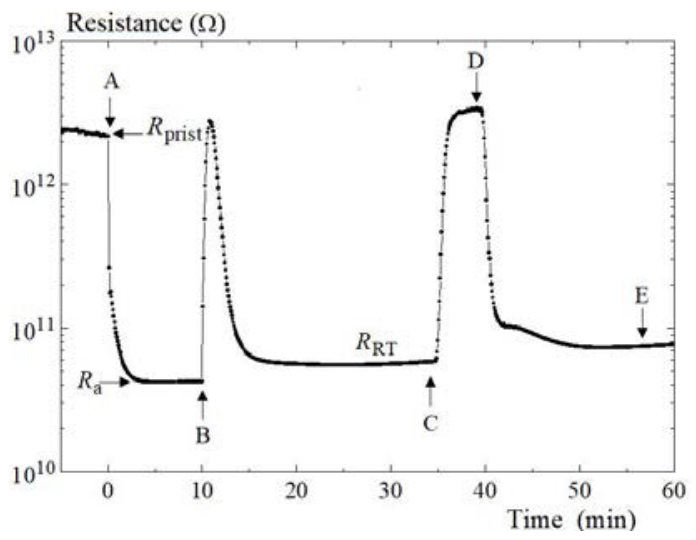

Fig. 2. Typical probing cycle consisting of the thermal pretreatment (from $A$ to $B$ ) and the response to acetone (from $C$ to $D$ )

the heating was achieved by means of the integrated resistive heater.

The sensing response was identified as the resistance measured between the interdigital electrodes upon the exposure of acetone vapors diluted in synthetic (dry) air ( $\left.R_{\text {acet }}\right)$ ratioed to the resistance obtained in pure synthetic air $\left(R_{\text {air }}\right)$. The resistance was standardly examined at constant operating voltage of $10 \mathrm{~V}$ applied across the interdigital electrodes. Varying acetone concentration was controlled by mixing of synthetic air and a certified mixture of 20 ppm acetone vapors in synthetic air (SIAD, Slovakia) by means of mass-flow controllers. Standardly, the total medium flow of $10 \mathrm{sccm}$ was kept constant for any (hence also zero) acetone concentration. The volume of the probing cell including supply pipes was about 30 $\mathrm{cm}^{3}$.

Raman spectra were collected at room temperature using a confocal Raman spectrometer (WITec Alpha300, Ulm, Germany) equipped with a laser operating at the wavelength of $531.93 \mathrm{~nm}$ (Spectra Physics Excelcior 53260 laser). The excitation power of the laser and the integration time for a spectrum were set to $1 \mathrm{~mW}$ and 10 min, respectively. The probed area and depth were about $500 \mathrm{~nm}$ (diameter) and $1 \mu \mathrm{m}$, respectively.

$\mathrm{X}$-ray photoemission spectra were recorded using a Thermo Scientific K-Alpha XPS system (Thermo Fisher Scientific, UK) equipped with a micro-focused, monochromatic Al K $\alpha$ a X-ray source $(1486.7 \mathrm{eV})$. An X-ray beam of $400 \mathrm{~mm}$ size was used at $6 \mathrm{~mA} \times 12 \mathrm{kV}$. The survey spectra were acquired in the constant analyzer energy mode with pass energy of $200 \mathrm{eV}$. Narrow regions were collected using the pass energy of $50 \mathrm{eV}$. Charge compensation was achieved with the system dual beam flood gun. The Thermo Scientific Avantage software (the version 5.9904, Thermo Fisher Scientific) was used for the digital acquisition and the data processing. Spectral calibration was determined by using the automated calibration routine and the internal $\mathrm{Au}, \mathrm{Ag}$ and $\mathrm{Cu}$ standards supplied with the K-Alpha system.

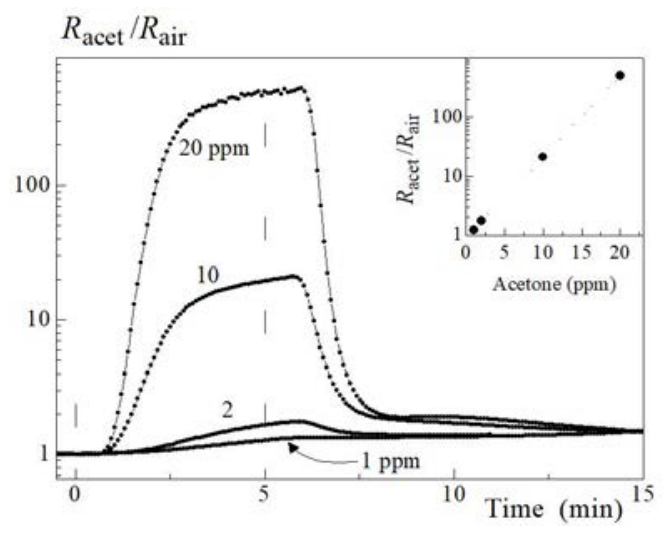

Fig. 3. Dynamic responses of the alumina to various concentrations of acetone vapours diluted in air (indicated in ppm units next to the dependences) recorded at $\mathrm{RT}$

\section{Results}

The alumina, which has been commonly employed in the role of the substrate, is supposedly inert to analytes. Indeed, we observed no sensitivity to acetone, yet, this has applied for the pristine alumina only. In contrast to the untreated alumina, the annealed alumina can display a marked response to acetone vapours. Figure 2 illustrates the resistance evolution of the alumina chip during the cycle consisted of the thermal pretreatment, cooling, and exposure to acetone vapors. The resistance of the pristine alumina, $R_{\text {prist }}$, plummets by almost two orders of magnitude to the value $R_{\mathrm{a}}$ upon the warming the alumina (the start is indicated by the label $A$ ) up to the annealing temperature (of $355^{\circ} \mathrm{C}$ here).

Upon the switching OFF the heater (indicated by $B$ ) the sample is brought near to RT in less than ten seconds, and the RT retains for the rest of the cycle, accordingly up to the label $E$. After the switching the heater OFF, the resistance at first increases near to $R_{\text {prist }}$, then it drops to a post-annealed value $R_{\mathrm{RT}}$, whilst the inequality $R_{\mathrm{a}}<R_{\mathrm{RT}}$ holds. The sample was kept in the dry-air flow of $10 \mathrm{sccm}$ during both the pretreatment and the cooling till to the label $C$. A mixture of $20 \mathrm{ppm}$ of acetone vapors diluted in the dry air (likewise with the flow of $10 \mathrm{sccm}$ ) was supplied during the 5 min interval between $C$ and $D$. The segment from $C$ to $E$ refers to the dynamic response curve (DRC). We remind that the sensing temperature, which refers to the temperature of the sensor while probing the dynamic response curve, was amounted to RT. The $R_{\text {acet }} / R_{\text {air }}$ ratio gives the pronounced value of about 60 . Note that - in reference to Fig. $2-R_{\text {air }}$ was identified with $R_{\mathrm{RT}}$ deduced at the time labeled $C$, while $R_{\text {acet }}$ was assigned to the maximal resistance achieved upon the acetone exposure, accordingly read at the label $D$.

The decreased/increased resistance of a chemiresistor upon the adsorption of an analyte is commonly referred to the $n$-/ $p$-type response and is often observed upon the adsorption of reducing/oxidizing species (eg CO, acetone/eg $\mathrm{NO}_{2}$ ), see $e g$ [3-4]. Referring to Fig. 2, the alu- 


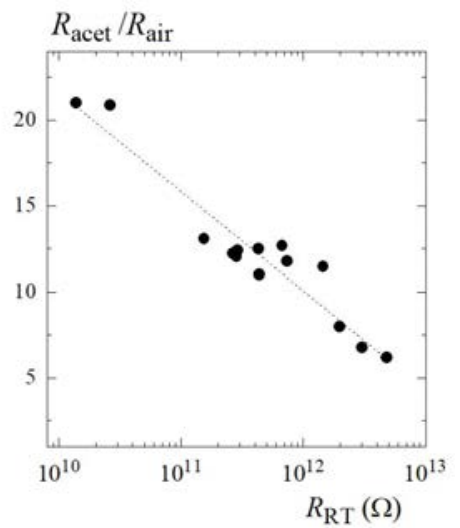

Fig. 4. $R_{\text {acet }} / R_{\text {air }}$ of alumina at RT upon the 5 min-exposure of $10 \mathrm{ppm}$ of acetone correlated with the resistance $R_{\mathrm{RT}}$ (see Fig. 2); the dashed line indicates the linear fit.

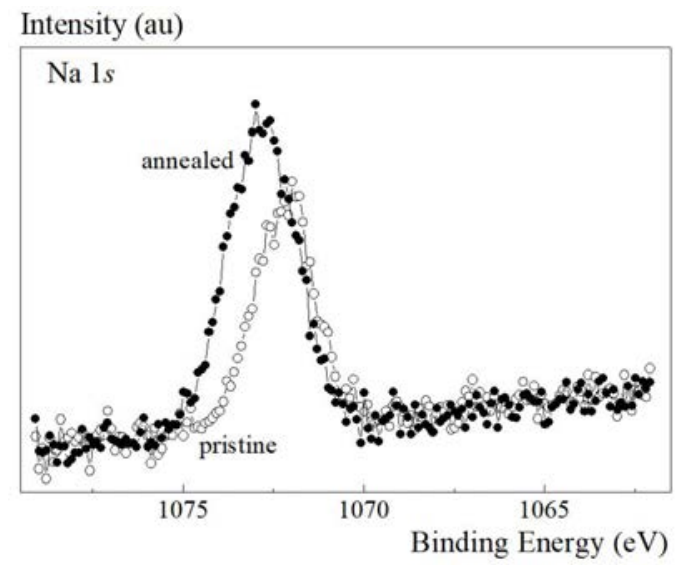

Fig. 6. $\mathrm{Na} 1 s$ core level of the $\alpha-\mathrm{Al}_{2} \mathrm{O}_{3}$ : the pristine sample (empty symbols) and 3 hours after the 15-min lasted annealing in dry air at $440^{\circ} \mathrm{C}$ (full symbols)

mina shows the $p$-type response to the acetone exposure, accordingly the trend is opposite to the generally assumed one, though exceptions have been already reported [3].

A certain delay of both the onset and decay of the dynamic response with the respect of the labels $C$ and $D$ corresponding to the switching $\mathrm{ON}$ and $\mathrm{OFF}$ of the acetone mixture supply, respectively, is due to a delay necessary for the exchange of gaseous ambient in the probe cell. The concentration change in the probe cell is not immediate upon the switching the probed mixture ON, but it approaches asymptotically to the adjusted value. Note that the volume of the probing cell including supply pipes matches with the 180 seconds-lasting gas flow of $10 \mathrm{sccm}$.

Figure 3 presents the dynamic response curves (DRC) of alumina to $1,2,10$, and $20 \mathrm{ppm}$ of acetone. Note that the response exceeding 500 was detected upon $20 \mathrm{ppm}$ of acetone. The inset shows the response $R_{\text {acet }} / R_{\text {air }}$ versus the acetone concentration dependence. The acetone exposures lasted $5 \mathrm{~min}$, the vertical dashed lines indicated switching $\mathrm{ON} / \mathrm{OFF}$ of the valve with the acetone mix-

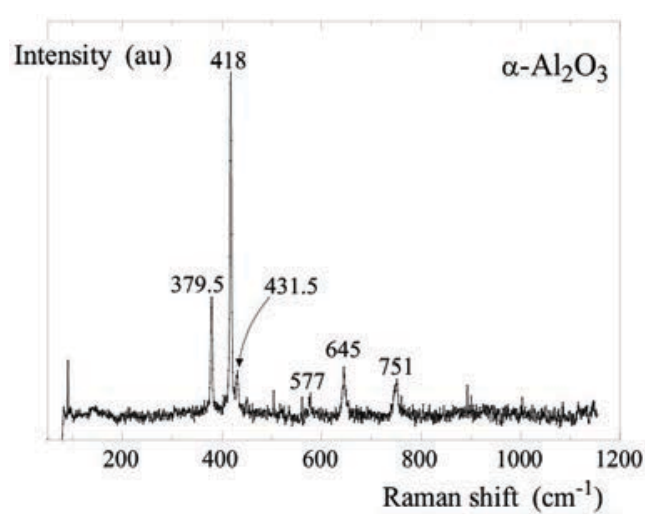

Fig. 5. Raman spectrum of the employed alumina, the constant background was subtracted.

ture. The $R_{\mathrm{RT}}$ 's for particular DRCs were in the interval $(1$ to 2.5$) \times 10^{10} \Omega$. The inset traces the response versus acetone concentration dependence extracted from the presented DRCs.

The response is reciprocally affected by the resistance $R_{\mathrm{RT}}$ (Fig. 4), which reflects the modification of alumina properties by the thermal pretreatment; specifically, both $R_{\mathrm{a}}$ and $R_{\mathrm{RT}}$ decreases with the increasing annealing temperature (not shown here). The dependence of the response $R_{\text {acet }} / R_{\text {air }}$ on $R_{\mathrm{RT}}$ examined upon the 10 ppm-acetone exposure suggests that the proportion $R_{\text {air }} / R_{\text {acet }} \propto \log \left(R_{\text {prist }}-R_{\mathrm{RT}}\right)$ holds.

The "sensing stage" of alumina - defined by the inequality $R_{\mathrm{RT}}<<R_{\text {prist }}$ here - is not stable at RT, as it is suggested by a slow gradual increase of $R_{\mathrm{RT}}$ at RT; as an example, $R_{\mathrm{RT}}$ increases from ca $5.55 \times 10^{10} \Omega$ to $5.7 \times 10^{10} \Omega$ in the course of $10 \mathrm{~min}$ (see the region of DRC afore the label $C$ in Fig. 2). The increasing $R_{\mathrm{RT}}$ (with the time elapsed after the annealing) results in the decreasing response as suggested by the dependence shown in Fig. 4. To eliminate the effect of the gradual relaxation of the pretreated alumina on the response, the same thermal treatment was performed immediately before probing each DRC shown in Fig. 3, thereby resulting in virtually the same $R_{\mathrm{RT}}$ at the beginning of each DRC characterization.

\section{Discussion}

The alumina is a common active element in humidity sensors [8]. Yet, we presume that the resistance decrease to $R_{\mathrm{RT}}$ after the thermal pretreatment is not explicable by means of humidity influence - though the water adsorption would admittedly result in the resistance decrease - since the entire cycle exemplified in Fig. 2 was carried out in dry synthetic air.

Gas-sensing properties of alumina-based films, though functionalized by eg metal or metal oxides additives, were reported [9-10]. Nevertheless, the insensitiveness the untreated alumina substrate to analytes had been tacitly 
presumed. The view was recently undermined in the study by Kita et al[11], where sensing responses to various gases - though at high concentrations - were demonstrated, such as NO (ca $500 \mathrm{ppm}$ ), $\mathrm{NO}_{2}$ (ca $100 \mathrm{ppm}$ ), $\mathrm{C}_{3} \mathrm{H}_{8}$ (ca $650 \mathrm{ppm}$ ), and $\mathrm{NH}_{3}$ (ca $50 \mathrm{ppm}$ ). It should be mentioned that our alumina samples showed virtually no response to $5 \mathrm{ppm}$ of $\mathrm{NO}_{2}$ neither at RT not at elevated temperature of $400^{\circ} \mathrm{C}$.

Here, the influence of the thermal pretreatment on alumina properties was investigated by Raman and x-ray photoemission spectroscopies. The observed vibrations in Raman spectrum (Fig. 5) indicate the $\alpha$ phase $[12,13]$. The spectra were insensitive to the annealing in dry air up to the investigated temperature of $440^{\circ} \mathrm{C}$. Compared to the $\delta, \theta$ and $\gamma$ phases of $\mathrm{Al}_{2} \mathrm{O}_{3}$, the $\alpha-\mathrm{Al}_{2} \mathrm{O}_{3}$ phase was reported to contain the highest concentration of sodium ions [13]. Indeed, the presence of sodium was verified in the pristine sample by the XPS method (the empty symbols in Fig. 6), namely the concentration of ca $0.5 \%$ was observed. Importantly, the $\mathrm{Na}$ concentration higher by a factor of about 1.6 was measured three hours after the annealing (the full symbols in Fig. 6) suggesting that the annealing induces the enrichment with sodium in the near-surface region. Besides, the elemental analysis revealed the presence of calcium and magnesium in the alumina. Calcium concentration being of about $0.5 \%$ was not affected by the annealing. Magnesium concentration showed the trend similar - yet less pronounced - to sodium concentration; namely, its concentration has arisen by about $15 \%$ due to the annealing.

Since the lesser relative changes of magnesium concentration compared to that of sodium, we presume that the surface properties of alumina are dominantly affected by sodium. The sensing mechanism itself is not fully understood so far, nevertheless, we propose a model as follows. The pre-annealing of alumina induces an agglomeration of $\mathrm{Na}^{+}$ions near the alumina surface (Fig. 6), which is manifested by the decrease of the room-temperature resistance $\left(R_{\mathrm{RT}}\right)$; we remind here that the alumina resistance was measured laterally. By this means modified alumina is effective in sensing the acetone. We recall that this state is not stable at RT as it is manifested by gradually increasing resistance towards the magnitude typical for the pristine sample in the course of tenths of hours. The resistance increase is owing to diffusion of the near-surface agglomerated sodium ions into the bulk of alumina. Upon the adsorption of acetone molecules, the near-surface agglomerated sodium atoms are presumably immobilized due to an interaction between the electron pair of the acetone molecule and the $\mathrm{Na}^{+}$ion thus forming an acid-basic pair in terms of Lewis theory [14]. The immobilization of the $\mathrm{Na}^{+}$ions entails the resistance increases, thereby demonstrating the $p$-type response. Unlike acetone adsorption, the adsorbed $\mathrm{NO}_{2}$ molecules do not virtually affect the alumina resistance. This is presumably due to a weaker interaction between the electron pair of $\mathrm{NO}_{2}$ molecule and $\mathrm{Na}^{+}$ion, as suggested by the markedly lower dipole moment of $0.316 \mathrm{D}$ of the former in comparison to that of acetone amounted to $2.88 \mathrm{D}$ [15], where $\mathrm{D}$ refers to the debye unit.

\section{Summary}

The resistance of the alumina wafer displays a high sensitivity to the exposure of acetone vapors in the ppm range at room temperature provided that a thermal pretreatment of the alumina is carried out prior the sensing characterization.

The thermal pretreatment enriches the surface region of alumina by sodium ions, originally distributed in the bulk of alumina. It is presumed that sodium ions are immobilized by interaction with acetone molecules causing the resistance increase upon acetone exposure, thereby the $p$-type response.

The stage with higher sodium concentration near the alumina surface, which means the condition susceptible for the acetone detection, slowly relaxes at room temperature towards the magnitude of the pristine sample. Nevertheless, the observed effect suggests that the alumina is applicable in capacity of a room temperature-operated sensor of acetone vapors.

\section{Acknowledgement}

This work was supported by Slovak National Grant Agency (grant number APVV-14-0891) and the project Efficient controlling of the production and consumption of energy from renewable sources (ITMS Code 26240220028) supported by the Research and Development Operational Programme funded by ERDF.

\section{REFERENCES}

[1] C. Deng, J. Zhang, X. Yu, W. Zhang, and X. Zhang, "Determination of acetone in human breath by gas chromatographymass spectrometry and solid-phase microextraction with on-fiber derivatization", Journal of Chromatography B, vol. 810, pp. 269-275, 2004.

[2] G.-T. Fan, C.-L. Yang, C.-H. Lin, C.-C. Chen, and C.-H. Shih, "Applications of Hadamard transform-gas chromatography/mass spectrometry to the detection of acetone in healthy human and diabetes mellitus patient breath", Talanta, vol. 120, pp. 386-390, 2014.

[3] J. Ivanco, S. Luby, M. Jergel, P. Siffalovic, M. Benkovicova, Y. Halahovets, E. Majková, R. Rella, and M. G. Manera, "Nitric Dioxide and Acetone Sensors Based on Iron Oxide Nanoparticles", Sensor Letters, vol. 11, pp. 2322-2326, 2013.

[4] S. Capone, M. G. Manera, A. Taurino, P. Siciliano, R. Rella, S. Luby, M. Benkovicova, P. Siffalovic, and E. Majkova, " $\mathrm{Fe}_{3} \mathrm{O}_{4} / \gamma$ $-\mathrm{Fe}_{2} \mathrm{O}_{3}$ nanoparticle multilayers deposited by the Langmuir -Blodgett technique for gas sensors application", Langmuir, vol. 30, pp. 1190-1197, 2014.

[5] Š. Luby, J. Ivančo, M. Jergel, P. J. Švec, M. Kotlár, D. Kostiuk, J. Halahovets, J. Kollár, J. Mosnáček, and E. Majková, "Thermal stability of $\gamma-\mathrm{Fe}_{2} \mathrm{O}_{3}$ nanoparticles and their employment for sensing of acetone vapours", IOP Conf. Series: J. Phys., vol. 939, pp. 012006-012009, 2017. 
[6] N. Padma, A. Joshi, A. Singh, S. K. Deshpande, D. K. Aswal, S. K. Gupta, and J. V. Yakhmi, " $\mathrm{NO}_{2}$ sensors with room temperature operation and long term stability using copper phthalocyanine thin films", Sensors and Actuators B: Chemical, vol. 143, pp. 246-252, 2009.

[7] M.-Y. Chuang, Y.-T. Lin, T.-W. Tung, L.-Y. Chang, H.-W. Zan, H.-F. Meng, C.-J. Lu, and Y.-T. Tao, "Room-temperature-operated organic-based acetone gas sensor for breath analysis", Sensors and Actuators B: Chemical, vol. 260, pp. 593-600, 2018.

[8] Z. Chen and C. Lu, "Humidity Sensors: A Review of Materials and Mechanisms", Sensor Letters, vol. 3, pp. 274-295, 2005.

[9] A. Mozalev, M. Bendova, R. M. Vazquez, Z. Pytlicek, E. Llobet, and J. Hubalek, "Formation and gas-sensing properties of a porous-alumina-assisted 3-D niobium-oxide nanofilm", Sensors and Actuators B: Chemical, vol. 229, pp. 587-598, 2016.

[10] T. Hyodo, T. Hashimoto, T. Ueda, O. Nakagoe, K. Kamada, T. Sasahara, S. Tanabe, and Y. Shimizu, "Adsorption/combustion -type VOC sensors employing mesoporous $\gamma$-alumina co-loaded with noble-metal and oxide", Sensors and Actuators B: Chemical, vol. 220, pp. 1091-1104, 2015.
[11] J. Kita, A. Engelbrecht, F. Schubert, A. Gro, F. Rettig, and R. Moos, "Some practical points to consider with respect to thermal conductivity and electrical resistivity of ceramic substrates for high-temperature gas sensors", Sensors and Actuators B: Chemical, vol. 13, pp. 541-546, 2015.

[12] P. G. Li, M. Lei, and W.H. Tang, "Raman and photoluminescence properties of $\alpha-\mathrm{Al}_{2} \mathrm{O}_{3}$ microcones with hierarchical and repetitive superstructure", Materials Letters, vol. 64, pp. 161-163, 2010.

[13] G. Deo, F. D. Hardcastle, M. Richards, A. M. Hirt, and I. E. Wachs, "Raman Spectroscopy of Vanadium Oxide Supported on Alumina", pp. 317-328, R.T.K. Baker and L.L. Murell (Eds.), Novel Materials in Heterogeneous Catalysis, American Chemical Society, 1990.

[14] P. C. Stair, "The concept of Lewis acids and bases applied to surfaces", J. Am. Chem. Soc., vol. 104, pp. 4044-4052, 1982.

[15] W. M. Haynes, "CRC Handbook of Chemistry and Physics 97th ed.", CRC Press, Boca Raton, 2016.

Received 19 March 2019 